\title{
ENU Mutagenesis Screen to Establish Motor Phenotypes in Wild-Type Mice and Modifiers of a Pre-Existing Motor Phenotype in Tau Mutant Mice
}

\author{
Xin Liu, ${ }^{1}$ Michael Dobbie, ${ }^{2}$ Rob Tunningley, ${ }^{2}$ Belinda Whittle, ${ }^{2}$ Yafei Zhang, ${ }^{2}$ \\ Lars M. Ittner, ${ }^{1}$ and Jürgen Götz ${ }^{1}$ \\ ${ }^{1}$ Alzheimer's and Parkinson's Disease Laboratory, Brain \& Mind Research Institute, University of Sydney, 100 Mallett Street, \\ Camperdown, NSW 2050, Australia \\ ${ }^{2}$ Australian Phenomics Facility, Australian National University, 117 Garran Road, Acton, ACT 0200, Australia
}

Correspondence should be addressed to Jürgen Götz, juergen.goetz@sydney.edu.au

Received 24 August 2011; Accepted 4 November 2011

Academic Editor: Kurt Bürki

Copyright (c) 2011 Xin Liu et al. This is an open access article distributed under the Creative Commons Attribution License, which permits unrestricted use, distribution, and reproduction in any medium, provided the original work is properly cited.

\begin{abstract}
Modifier screening is a powerful genetic tool. While not widely used in the vertebrate system, we applied these tools to transgenic mouse strains that recapitulate key aspects of Alzheimer's disease (AD), such as tau-expressing mice. These are characterized by a robust pathology including both motor and memory impairment. The phenotype can be modulated by ENU mutagenesis, which results in novel mutant mouse strains and allows identifying the underlying gene/mutation. Here we discuss this strategy in detail. We firstly obtained pedigrees that modify the tau-related motor phenotype, with mapping ongoing. We further obtained transgene-independent motor pedigrees: (i) hyperactive, circling ENU 37 mice with a causal mutation in the Tbx1 gene-the complete knock-out of Tbx1 models DiGeorge Syndrome; (ii) ENU12/301 mice that show sudden jerky movements and tremor constantly; they have a causal mutation in the Kcnq1 gene, modelling aspects of the Romano-Ward and Jervell and Lange-Nielsen syndromes; and (iii) ENU16/069 mice with tremor and hypermetric gait that have a causal mutation in the Mpz (Myelin Protein Zero) gene, modelling Charcot-Marie-Tooth disease type 1 (CMT1B). Together, we provide evidence for a real potential of an ENU mutagenesis to dissect motor functions in wild-type and tau mutant mice.
\end{abstract}

\section{Introduction}

With an ageing population, Alzheimer's disease (AD) and related neurodegenerative disorders represent a serious social and economic threat to most societies. The cost of caring for an increasing number of people with dementia continues to rise. At present, there are an estimated 30 million people with dementia worldwide, with numbers expected to further increase to 80 million by 2040 . The countries or regions with the largest numbers of affected individuals are China and the developing western Pacific, Western Europe, and the Unites States [1]. The two core histopathological hallmarks of $\mathrm{AD}$ are the amyloid- $\beta(\mathrm{A} \beta)$ plaques and the tau-containing neurofibrillary tangles (NFTs), both of which have been described by Alois Alzheimer more than a century ago [2]. Disease-caus-ing mutations in familial cases of $\mathrm{AD}$ have been described two decades ago; yet there is still no cure for this debilitating disease. The current treatment is limited to acetylcholine esterase (AChE) inhibitors (donepezil, rivastigmine, and gal-antamine) and the NMDA receptor antagonist, memantine; yet neither of these strategies halts the degenerative process that characterizes $\mathrm{AD}[3]$.

What are the characteristics of the major component of the two hallmark lesions in $\mathrm{AD}$ and what causes the familial cases? The 39-42 amino acid $A \beta$ peptide in the plaques is fibrillar, as is tau, a microtubule-associated protein that becomes hyperphosphorylated in the disease process and eventually aggregates to form NFTs, neuropil threads, and the abnormal neurites that are associated with some amyloid- $\beta$ plaques [4]. Although tau has been generally perceived as a neuronal protein, with mainly an axonal localization, we have recently challenged this view by demonstrating an essential dendritic function under physiological conditions and in mediating $\mathrm{A} \beta$ toxicity $[5,6]$. In addition 
to plaques and NFTs, the $\mathrm{AD}$ brain is characterized by massive neuronal cell and synapse loss [7]. Tau pathology, in the absence of overt $A \beta$ plaques, characterizes a series of diseases that are collectively known as tauopathies. This includes FTLD-Tau (frontotemporal lobar degeneration with tau inclusions) with an inherited form known as FTDP17 (frontotemporal dementia with Parkinsonism linked to chromosome 17). There is a second, major subset of FTLD characterized by tau-negative yet ubiquitin-positive lesions. In this subset, the transcription and splicing factor TDP-43 (TAR DNA-binding protein 43) has been identified as the aggregating protein, and consequently, this form of FTLD has been named FTLD-TDP [8]. Similar to tau, TDP-43 in the aggregates is hyperphosphorylated and fragmented, a process believed to be linked to toxicity [8-11]. Finally, in a third subset of FTLD, FTLD-FUS, the nuclear protein FUS (Fusedin-Sarcoma) has been identified as aggregated protein [12]. While both proteins form also aggregates in subsets of amyotrophic lateral sclerosis (ALS), the mechanisms causing disease however are believed to differ [13].

The majority of $\mathrm{AD}$ cases are sporadic (SAD), with familial (FAD) cases likely accounting for less than $1 \%$. In three genes, autosomal dominant mutations have been identified: amyloid precursor protein (APP), presenilin 1 (PSEN1) as well as presenilin 2 (PSEN2) [14]. It is the amyloid precursor protein, APP, from which the $\mathrm{A} \beta$ peptide is derived by proteolytic cleavage. Presenilins are components of the multimeric $\gamma$-secretase complex that generates $\mathrm{A} \beta$. In addi-tion to the three FAD genes, apolipoprotein E (APOE) has been unanimously confirmed as a susceptibility gene in spo-radic $\mathrm{AD}$ (SAD) [14]. More recently, additional risk factor genes have been identified, with CLU encoding clusterin, PICALM the phosphatidylinositol-binding clathrin assembly protein, CR1 the complement component $(3 \mathrm{~b} / 4 \mathrm{~b})$ receptor 1 , and TOMM40 a channel-forming subunit of the translocase of the mitochondrial outer membrane (TOM) complex [1517]. At present, a total of ten genes, mostly with roles in the immune system and in cholesterol metabolism, are established risk genes for $\mathrm{AD}$ [18].

Compared to AD, FTLD is a more heterogeneous disorder, with variants that are, for example, characterized by language or behavioural problems [19]. AD and FTLD both present with a progressive decline of memory function leading to dementia, although in FTLD this is often preceded by motor symptoms such as Parkinsonism [20]. It was several years after the first mutations had been identified in the $A P P$ and PSEN genes in $\mathrm{AD}$, that the first mutations were identified in a subset of FTLD-Tau, FTDP-17, where they were found in exonic and intronic sequences of the MAPT gene that encodes tau [21]. Another subset of familial FTLD is characterized by mutations in the PGRN gene that encodes the pleiotropic protein progranulin, in the VCP gene that encodes valosin-containing protein, and also in TARDP encoding TDP-43 [19]; these FTLD-TDP cases are characterized by inclusions of TDP-43 [8]. Finally, mutations in $C H M P 2 B$ encoding chromatin modifying protein $2 \mathrm{~B}$ lead to FTLD, in the absence of either tau or TDP-43 inclusions [21].

In this paper, we will be discussing a few selected tau transgenic mouse models with memory and motor phenotypes and how one of these, the K369I mutant human tau expressing K3 strain [22], has been employed in an ENU modifier screen [23], outlining the strategy from breeding to gene identification.

\section{Tau Transgenic Mouse Models with Memory and Motor Phenotypes}

As the focus of this review article is the application of ENU mutagenesis to one of our mutant tau transgenic mouse strains, K3, we will be comparing this particular strain with a few other tau transgenic strains we have established in the past. Mouse strains that are otherwise available in the field including those characterized by $\mathrm{A} \beta$ plaque formation have been discussed by us in detail elsewhere [24-27].

What has the ambition been in developing transgenic mouse strains for the tau pathology of $\mathrm{AD}$ in the first place? The aim has always been to authentically model the human pathology and to gain a deeper understanding of what causes tau pathology, how this is related to neurodegeneration and functional impairment, and ultimately, how pathogenic processes can be prevented or delayed. When we generated the very first tau transgenic mouse model in 1995 by expressing the longest human tau isoform in mice, we reproduced a major aspect of the human pathology in a subset of neurons: somatodendritic accumulation of hyperphosphorylated forms of tau, a feature of pathological tau in AD brains [28]. We failed however to reproduce NFT formation, neurodegeneration, and functional impairment. Even by using a stronger promoter to drive human tau expression, this goal of the early days was not achieved, although the mice developed a motor phenotype and amyotrophy $[29,30]$.

Only with the identification of pathogenic mutations in FTLD-Tau in the MAPT gene encoding tau, by expressing mutant forms such as G272V or P301L tau, we achieved NFT formation, as determined by Gallyas silver impregnations and electron microscopy [31,32]. While we discontinued the G272V mutant mice [32], the P301L tau transgenic pR5 strain was employed to provide support for the amyloid cascade hypothesis that places $A \beta$ upstream of tau [33], to reveal behavioural impairments in amygdala- and hippocampus-dependent tasks $[34,35]$, to assess the role of site-specific phosphorylation and in particular of protein phosphatase $2 \mathrm{~A}(\mathrm{PP} 2 \mathrm{~A})$ in this process $[36,37]$, to determine the degree of the cholinergic pathology [38], and to reveal an impaired stress-related unfolded protein response [39] and a mitochondrial dysfunction caused by pathological tau [4042]. Assessing the role of miRNAs in AD adds an additional level of complexity $[43,44]$.

While the pR5 mice display memory impairment as a major clinical feature of $\mathrm{AD}$, another feature, Parkinsonism, that characterizes a significant subset of FTLD cases, has been modelled in K369I mutant tau transgenic K3 mice. We established this strain based on the identification of the K369I mutation of tau in a single patient with Pick's disease (PiD), a form of FTLD [45]. As is known for PiD in general, the tau lesions in the mutation carrier's brain showed a remarkable feature when analysed histologically: silver impregnation 
revealed that the tau lesions were Bielschowsky-positive and Gallyas-negative; moreover, tau was phosphorylated at many epitopes but not 12E8 (Ser262/Ser356). We succeeded in reproducing this distinct pathology fully in the $\mathrm{K} 3$ mice that express K369I mutant tau [22]. Memory functions were impaired as shown in the novel object recognition test. Owing to a unique expression pattern of the K369I transgene that includes the substantia nigra, the $\mathrm{K} 3$ transgenic mice also modelled early-onset Parkinsonism, that is, resting tremor, bradykinesia, postural instability, and gait anomalies. They showed an increased cataleptic response to haloperidol and an early, but not late, response to L-Dopa, indicating that the dopaminergic system is impaired in these mice. In recent years, impaired axonal transport has been identified as a central pathomechanism in $\mathrm{AD}$ [46-51]. Conceptually, impaired axonal transport is linked to oxidative stress and mitochondrial dysfunction [52]: mitochondria need to be efficiently transported along the long axonal processes for neurons to function; furthermore they are a major source of reactive oxygen species (ROS) [53, 54]. We found a selectively impaired axonal transport of distinct cargos including mitochondria and TH- (tyrosine hydroxylase-) containing vesicles [22]. At the molecular level, we found that this impaired transport phenotype is due to trapping of a component of the kinesin motor machinery, the adapter protein JIP1, by elevated levels of phosphorylated tau, preventing JIP1 from executing its physiological function in the axon. A pathological interaction between tau and JIP1 was further revealed in $\mathrm{AD}$ and not, control brain, highlighting the validity of transgenic animal models in dissecting pathomechanisms in AD [55]. As tau only traps JIP1 when it is hyperphosphorylated, this study presents inhibition of abnormal hyperphosphorylation of tau as a promising therapeutic strategy in $\mathrm{AD}[56]$.

\section{Modulation of Transgenic Phenotype by Therapeutic Intervention}

With the pR5 and the K3 mice we do have two complementary models at hand that present themselves for therapeutic intervention: both strains are characterized by hyperphosphorylation and increased insolubility of tau as well as silver-positive inclusions, Gallyas-positive NFTs in the case of R5 mice [33], and Bielschowsky-positive inclusions predominantly resembling Pick bodies in K3 mice [22]. The pR5 mice show an accelerated extinction in the conditioned taste aversion (CTA) paradigm [34], while the K3 mice have a memory phenotype as determined by the novel object recognition test, as well as a motor phenotype that can be easily monitored either by scoring of clasping, gait, and tremor (Table 1 ) or on the Rota-Rod $[22,57]$. In addition, as the $\mathrm{K} 3$ mice age, they lose substantial numbers of neurons both in the substantia nigra (TH-positive neurons) and in the cerebellum (Basket cells) [22, 57]. Experimental diabetes exacerbates the tau pathology as shown for pR5 mice [58].

As therapeutic strategies, different approaches can be envisaged such as the transplantation of cells with the potential to differentiate in situ either into neuronal or glial cell types [59]. This strategy has been successfully applied to mice with a combined tau and $A \beta$ pathology in which neuronal stem cell transplantation has been shown to improve cognition via brain-derived neurotrophic factor (BDNF) [60]. Other strategies that are recently pursued are tau-based immunizations [61-64]. We used both active and passive immunization and monitored efficacy of this approach biochemically, histologically, and behaviourally [65]

We have also been testing a small bioavailable compound, sodium selenate that can be supplied with the drinking water. Chronic treatment over a period of four months significantly improved the pathology in both young and old K3 mice. The mice showed a gradual improvement on the Rota-Rod [57]. This was associated with reduced tau hyperphosphorylation and decreased numbers of tau inclusions including spheroids. In fact, spheroids were completely absent from frontal cortex, suggesting that selenate reverts the functional impairment leading to spheroids. K3 mice are characterized by a substantial, age-dependent degeneration of cerebellar basket neurons, resulting in the absence of pinceau terminals formed by clustered axons surrounding Purkinje cells. Selenate treatment fully prevented this degeneration as well. Taken together, these findings show that selenate reduces tau phosphorylation and deposition, mitigates pathological spheroid formation, and prevents axonal degeneration of distinct neuronal populations in $\mathrm{K} 3$ mice.

We also treated 8-month-old pR5 mice with sodium selenate and found that treatment caused a significant impairment in the CTA paradigm. As for the K3 mice, selenate both caused reduced hyperphosphorylation and numbers in tau deposits (NFTs) as well as increased tau solubility. NFT numbers in 12-month-old selenate-treated pR5 mice were similar to those in 8-month-old-untreated pR5 mice, suggesting that treatment had halted disease progression. We further showed that the selenate-induced improvements require protein phosphatase $2 \mathrm{~A}$ (PP2A) [57], by performing in vitro experiments and showing inefficacy of selenate in mice with a combined tau pathology and reductions in PP2A activity $[66,67]$.

\section{ENU Mutagenesis Strategies in Mice}

While in the $\mathrm{AD}$ and ageing field mouse strains are available with traits that arose spontaneously and have been further established by specific breeding programs such as the senescence-accelerated SAM mice [68], the rate at which new models arise spontaneously means that, even with a concerted effort, discovery is on an asymptomatic trajectory towards a phenotype gap [69]. An alternative is random chemical mutagenesis [69]. The concept of genomewide mutagenesis is well established in invertebrates such as the fruit fly Drosophila melanogaster and in the nematode worm Caenorhabditis elegans, with ENU mutagenesis mainly producing $\mathrm{A}$ to $\mathrm{T}$ and $\mathrm{T}$ to $\mathrm{A}$ transversions [70]. This $\mathrm{mu}-$ tagenesis strategy was used to create the first genetic maps of Mendelian inheritance in Drosophila, revealing many of the key genes controlling early embryonic patterning and development [71]. What makes it possible to carry out comparable efforts in mice is the extraordinary mutagenic 
efficiency of ENU in mouse spermatogonial stem cells [72]. By expanding the library of alleles with chemical mutagenesis using N-ethyl-N-nitosourea (ENU), identification of each of the single nucleotide changes that result in a particular phenotype is feasible in mice and is now providing novel insights into not only immunity, where this approach has been used widely [73], but also the functioning of the brain [74].

Genomewide, random mutagenesis with the ethylating chemical ENU provides a way to produce and screen genetic variants in the mouse. Intraperitoneal injection of male founder mice (G0) causes random base pair substitutions in the DNA of spermatogonial stem cells. Informative mutations are identified by screening members of the pedigrees propagated from these founders for phenotypes of interest. Introducing point mutations has the advantage that it most often inactivates or alters the function of individual protein domains rather than eliminating the protein altogether (as is the case for complete knock-outs), making it harder for related proteins to compensate, better mimicking the effects of drugs or natural genetic variants, illuminating active sites in proteins, and revealing specific functions of alternatively spliced forms [23]. We have been investigating a phenotype modulation of our K3 mice by crossing ENU male founder mice with $\mathrm{K} 3$ females as discussed hereinafter. Here, and in general, dominant mutations are expected to manifest in the first generation offspring (G1), while recessive mutations are brought to homozygosity in G3 mice by either G1 × G2 or G2 × G2 crosses [69, 75-80]. The rate of recessive mutations has been established based on genotype-driven projects using libraries of G1 gametes generated from an ENU-treated founder. Sequencing of sentinel regions of the genome of G1 sperm provides quantitative and qualitative characteristics of ENU mutagenesis and information about the probable yield of mutations from phenotype-driven screens. The consensus is a mutation rate of $0.5-1.0$ mutations/Mbp $(7.5 \times 10-7$ mutations/bp/G0 gamete, or 1500-3000 mutations/G1) from a standard regimen $(100 \mathrm{mg} / \mathrm{kg} /$ week $\times 3$ for a C57BL/6 founder) [69]. Assuming a $1600 \mathrm{cM} 2.6 \mathrm{~Gb}$ haploid mouse genome, of which approximately $1.5 \%$ is coding, this comes down to approximately 50 functional mutations in each G1. Of these, four will be transmitted to homozygosity in each G3. The efficiency of ENU-induced mutation means that the generation of allelic series is highly feasible. Based on a conservative estimate of 20 point mutations per G1 gamete, a survey of 17400 G3s would yield a series of 5 functional alleles per gene with $>99 \%$ probability [69].

Limiting factors of the ENU mutagenesis approach are infertility and lethality, with the standard ENU regimen resulting in azoospermia (absence of motile sperm in semen) for up to 10 weeks, with fertility being resumed when the testes are repopulated by spermatogonia [81]. Persistent infertility on the other hand might occur as a direct consequence of mutagenesis [77]. The presence of mutations that confer lethality before weaning sets the workable upper limit of the ENU dose even more stringently. For example, one study found that $1 / 13 \mathrm{G} 1$ mice contained lethal mutations in the balancer chromosome 11 region that contains $5 \%$ of the genome, with the consequence that $18 \%$ of the G3 population will not survive because of lethal mutations [77].

For phenotype-driven strategies (such as a motor phenotype, see the following), F1 and F3 mice are examined for dominant-acting and recessive-acting mutations, respectively $[23,82]$. The recovery rate of dominant mutations is low $(2 \%)$ [83]; on the other hand however, costs for identifying recessive mutations are much higher, due to much higher mouse numbers and sample size to be screened and sequenced. In an ideal screen, the phenotypes are qualtitative (such as having a motor phenotype) or binomial (rejection of transplanted tissue or not); they are free of false positives and are sufficiently penetrant to permit meiotic mapping. The latter is typically achieved first by outcrossing and then an F1 intercrossing to yield F2 mice; 50 F2 mice are required to obtain a $10 \mathrm{Mb}$ mapping resolution with a $95 \%$ confidence [84], although haplotype mapping might enable an estimation of the chromosomal location using as few as 10 affected F2 mice [85]. For a more detailed discussion of the likelihood of a confounding mutation cosegregating with the phenotype the reader is referred to two excellent reviews on the topic $[23,69]$.

\section{ENU Mutagenesis Applied to K3 Mice Identifies Transgene-Dependent and-Independent Pedigrees}

Applying ENU mutagenesis to mice with neurological phenotypes is quite novel. What makes the approach feasible is a simple read-out, and in this respect, motor phenotypes are much easier to monitor than memory phenotypes. As in our K3 mice, K369I mutant tau expression causes both a motor and memory impairment [22], changes to the motor phenotype by any intervention although interesting in itself can also serve as a surrogate marker for changes in memory functions. We initiated an ENU mutagenesis program in K3 mice that generated transgene-dependent and -independent pedigrees. In the following we will be discussing five pedigrees, ENU164 (partial rescue, transgene-related), ENU67 (slow weight gain, transgene-related), ENU37 (circling, not transgene-related), ENU12/30a (motor phenotype, not transgene-related), and ENU16/069 (motor phenotype, not transgene-related).

To first establish that the K3 phenotype is fully penetrant on the background chosen for ENU mutagenesis, we established backcrosses with 20 mice each, using C57BL/10, $\mathrm{C} 3 \mathrm{H} / \mathrm{H} 3 \mathrm{H}, \mathrm{FVB}, \mathrm{C} 57 \mathrm{Led}$, and BALB/b mice, all of which are established ENU strains at the APF (Australian Phenomics Facility, Canberra). We decided to use BALB/b as the strain for ENU mutagenesis, as the K3 phenotype of Parkinsonism is fully penetrant on this background, both with regards to phenotype and age-of-onset. Therefore, BALB/b males were injected with ENU and paired with the K3 mice (that are on a C57BL/6 background), and the F1 offspring was analyzed. The litters born were scored for three read-outs of Parkinsonism in mice, tremor (staging 0-3), gait anomalies (staging 0-3), limb clasping (staging 0-5), as well as body weight (Table 1). 
TABLE 1: Phenotyping protocol for ENU/K3 mice. Four phenotypes are being scored, limb clasping, tremor, and gait abnormalities (cardinal features defining Parkinsonism) and body weight.

\section{(1) Limb clasping}

Procedure: $\rightarrow$ Lift the mice by the tail just so much above the grid, that they try to reach it with their front paws. Keep the mice in this position for at least 30 seconds. Should the phenotype not be obvious, touch hind limbs? This will ensure that clasping becomes obvious

\begin{tabular}{ll} 
Stage & Definition \\
0 & No clasping (wild-type phenotype) \\
1 & Short and transient clasping only after stimulation (grabbing of grid or touching of hind limbs) \\
2 & Persistent hind limb clasping after stimulation (grabbing of grid or touching of hind limbs) \\
3 & Spontaneous hind limb clasping without major stimulation (when lifted for a few seconds by the tail) \\
4 & Spontaneous clasping of hind- and fore limbs \\
5 & Clasping of hind- or fore limbs persists for more than $5 \mathrm{sec}$ after mice have been placed back into the home cage \\
\hline
\end{tabular}

(2) Tremor

Procedure: $\rightarrow$ Observe mice in home cage

\begin{tabular}{ll}
\hline Stage & Definition \\
0 & No tremor (wild-type) \\
1 & Intermitted mild tremor \\
2 & Continuous mild tremor \\
3 & Continuous intensive tremor \\
\hline
\end{tabular}

(3) Gait anomalies

Procedure: $\rightarrow$ Place mice on metal surface in the sterile hood. Ensure that surface between cages is clean. Observe gait and posture

\begin{tabular}{ll}
\hline Stage & Definition \\
0 & Normal gait, normal posture (wild-type) \\
1 & Intermittendly shaking gait, normal posture \\
2 & Shaking gait, spreading of hind limbs, flattening of the rear body part \\
3 & Continuously shaking gait, spreading of hind limbs, postural instability, dragging of rear body \\
\hline
\end{tabular}

(4) Body weight

Procedure: $\rightarrow$ Weigh mouse on the same balance in the same container. Record two digits after the comma

Schedule

Procedure: $\rightarrow$ First analysis at 4 weeks of age, with follow-ups every week until $x$ weeks of age

We had previously phenotyped wild-type BALB/b mice for the three Parkinsonism characteristics and weight. Although none of them displayed abnormal gait or resting tremor, 37\% did clasp their hind limbs when stimulated and $5 \%$ clasped their hind limbs without stimulation, while their front paws grasped the cage wire. Therefore, to ensure accurate identification of the phenotype, the hindlimb clasping alone was not used as sole indicator, but in conjunction with tremor and abnormal gait. Genotyping was done by PCR. Phenotyping was done at weaning, and then at oneweek intervals until around 8 weeks of age, depending on the scoring outcome. Any mice exhibiting all three phenotypes and genotyped K3-tau positive were culled, as were any mice failing to display any of the phenotypes and genotyped K3-tau negative. Mice which showed a genotype/phenotype mismatch were genotyped a second time for confirmation and phenotyped for up to 3 months. Any mice which did not display the phenotype until after 5 weeks of age were also noted as having delayed onset of the phenotype and were flagged as a putant (potential mutant) of interest. Putants were then bred to F3 by crossing the ENU strains to B6 twice. This was followed by Next Generation whole exome sequencing as a means to identifying mutations.
We would like to make the comment that while, previously, we have been mapping the ENU mutations down to a chromosomal region for most strains these days it is much faster to directly "next generation sequence" the exomes of one to two affected mice instead. This has greatly accelerated the speed of discovery from years to months and reduced costs of identifying causal mutations. For a recent review on next generation sequencing (NGS) see [86]. The SNPs identified with the sequencing were then validated against the phenotype with an Amplifluor SNP assay for each SNP. This identified only the causal mutation as it directly relates to the phenotype.

Hereinafter we will be discussing five pedigrees for which we have arrived at different stages of out-crossing and identification of the underlying gene mutations, starting with those pedigrees with which we have the least progressed.

ENU164 Pedigree. This pedigree is characterized by a partial rescue of the Parkinsonism phenotype that characterizes the $\mathrm{K} 3$ mice, because some transgene-positive mice in the pedigree showed an improvement in our vigorous weekly scorings; for example, at week 6 they would show a scoring of $1 / 2 / 1$ for gait/clasp/tremor instead of $3 / 4 / 3$ as is typical for 
K3 mice. As mentioned above, consecutive backcrossing onto C57BL/6 reduces the number of "functional" ENU-induced point mutations such that in the 3rd generation (F3) they are reduced to 3-4 mutations. As the $\mathrm{K} 3$ mice have been generated on a C57BL/6 background, while ENU mutagenesis has been on a BALB/b inbred genetic background, strainspecific SNP detection enabled the genomic mapping of the causative mutation location on the BALB/b background. This narrows down the linked mutation-carrying region to less than $20 \mathrm{Mb}$, at which point candidate genes in the region will be sequenced to detect mutant alleles, and eventually, the causative mutation. So far, we screened $39 \mathrm{~K} 3$-positive F2s obtained from Founder F1.164. 6 of these showed the inherited partial rescue phenotype ( $\sim 15 \%$ inheritance). From F2 \#483 we screened 11 K3-positive mice, with 3 showing partial rescue ( $\sim 27 \%)$, from F2 \#430 $20 \mathrm{~K} 3$-positive mice $(\sim 35 \%)$. Exome (NGS) next generation sequencing is ongoing to identify the one single gene mutation that causes the K3 modulating phenotype.

ENU67 Pedigree. This pedigree shows a worsening of the weight phenotype that characterises the K3 mice. For example, while the average weight of transgene-negative mice is 19,0 grams at six weeks of age, this is reduced to 15.8 grams for transgene-positive $\mathrm{K} 3$ mice and is even further reduced in the ENU67 pedigree (K3-positive) that shows an average weight of only 11.7 grams. F1.67 had 4 offspring, with 2 K3-positive mice. Both K3-positive mice (\#347, \#350) were identified as slow to gain weight, and we are currently crossing further to determine the underlying gene mutation.

ENU37 Pedigree. These mice are hyperactive and display an intermittent circling in both directions. Some of the mice show head tilting and weaving. The trait shows Mendelian inheritance over three generations. Following deep sequencing of the exome of 2 affected mice we identified possible causal point mutations in the following genes:

(i) 5730455P16Rik (RIKEN cDNA);

(ii) tcof1 (Treacher Collins Franceschetti syndrome 1), responsible for the production of treacle, which plays a role in the formation of tissue in the face. The human disease is also autosomal dominant;

(iii) Tbx1 (T-Box 1 gene) $T$-box genes encode transcription factors involved in the regulation of developmental processes. Chromosomal deletions in this region have been linked to neural-crest-related defects. A point mutation could potentially cause a mild neural phenotype;

(iv) Senp7 (SUMO1/sentrin specific peptidase 7 gene) involved in the posttranslational modification of proteins by the addition of ubiquitin-like SUMO proteins;

(v) Ggt7 (gamma-glutamyltransferase 7 gene): this gene is a member of a family that encodes enzymes involved in both the metabolism of glutathione and in the transpeptidation of amino acids; changes in the activity of $\gamma$-glutamyltransferase may signal preneoplastic or toxic conditions in the liver or kidney in humans;

(vi) Hkdc1 (hexokinase domain containing 1 gene);

(vii) Chrna4 (cholinergic receptor, nicotinic, alpha polypeptide 4 Gene): this gene encodes a nicotinic acetylcholine receptor. Mutations cause nocturnal frontal lobe epilepsy type 1 . Polymorphisms in this gene provide protection against nicotine addiction in humans.

Amplifluor primers were designed to the above SNPs and affected and unaffected mice genotyped, which led to the confirmation of the causal mutation in the $T b x 1$ gene, with a $\mathrm{T}$ to $\mathrm{A}$ substitution of bp 857 (coding position) resulting in an Asn to Ile change of amino acid 276. A complete knock-out of Tbx1 is available and is a model of DiGeorge Syndrome; it is further characterized by perinatal death $[87,88]$. The DiGeorge syndrome is caused by a 1.5 to $3.0 \mathrm{Mb}$ hemizygous deletion of chromosome 22q11.2. Haploinsufficiency of the $T b x 1$ gene in particular is responsible for most of the physical malformations. There is evidence that point mutations in the TBX1 gene can also cause the disorder. DiGeorge syndrome is the most frequent microdeletion syndrome in humans, and is characterized by cardiovascular, thymic and parathyroid, and craniofacial anomalies. The underlying cause is disturbed formation of the pharyngeal apparatus, a transient structure present during vertebrate development that gives rise to endocrine glands, craniofacial tissue, and the cardiac outflow tract. The pharyngeal apparatus is composed of derivatives of ectoderm, endoderm, mesoderm, and the neural crest. Thus, complex interactions between cell types from different origins have to be orchestrated in the correct spatiotemporal manner to establish proper formation of the pharyngeal system. The analysis of engineered mouse mutants developing a phenotype resembling DiGeorge syndrome has revealed genes and signalling pathways crucial for this process. Intriguingly, these mouse models reveal that interference with either of two distinct phases of pharyngeal apparatus development can contribute to the aetiology of DiGeorge syndrome [89]. With regards to AD, Tbx1 has been shown to be upregulated when mice are injected with $\mathrm{A} \beta[90]$. Furthermore, String 9.0 Known and Predicted Protein-Protein Interactions for Tbx1 identify Sept5 which is also among 29 genes that are deregulated in pR5 tau transgenic mice (JG and LIM, manuscript submitted). The value of the ENU37 strain lies in the absence of a perinatal phenotype which allows for studies which are not feasible with the complete knockout.

ENU12/301 Pedigree (in Short: ENU12). ENU12 mice, under mild stress, show sudden jerky movements when moving around the cage and appear to tremor constantly. They often toss their heads upwards. Some mice show what could be described as opisthotonus (a type of spasm in which the head and heels arch backwards in extreme hyperextension and the body forms a reverse bow). The mice display varying degrees of severity; even when severely affected, they are able to move 
freely and quickly around the cage. They occasionally fall to either side and circle around the cage in very fast movements when highly stressed, such as when the cage is changed. There is no preference in a single mouse to circle in either direction. The ENU12 phenotype can be recognised at weaning.

Deep sequencing identified an $\mathrm{A}$ to $\mathrm{T}$ substitution of bp 1408 in exon 10 of the Kcnq1 gene that results in a Lys to STOP change of amino acid 435. Aliases of KCNQ1 are KCNA9, KVLQT1, AW559127, and Kv7.1. The KCNQ1 gene encodes a voltage-gated potassium channel protein required for the repolarisation phase of the cardiac action potential. The gene product can form multimers with two other potassium channel proteins, KCNE1 and KCNE3. In-herited forms of a human condition known as Long QT syn-drome (LQTS), an abnormality in the cardiac ventricular repolarisation characterised by QT interval prolongation and abnormal T-waves on an electrocardiogram (ECG), are most commonly associated with mutations in KCQN1. Some patients show only a cardiac defect (Romano-Ward sy-ndrome, RWS, dominant); others may suffer from severe deafness due to a lack of endolymph in the vestibular and auditory compartment (Jervell and Lange-Nielsen syndrome, JLNS, recessive) [91].

The complete knockout is a model of JNLS [92], but the mice show an additional circling (shaker/waltzer) and rapid head blobbing phenotype. Introducing the familial A340E point mutation by a knock-in approach models RWS (cardiac phenotype, but no inner ear defect) [93]. This point mutation is in the amino-terminus of KCNQ1 that contains the six transmembrane domains S1-S6 and the pore domain P [94]. A comparative analysis of the strains allowed the authors to draw conclusions with regards to the etiology of the cardiac phenotype. How does the ENU12 strain differ from the above two models? The K434Stop mutation in the ENU12 mice is in the carboxy-terminus that contains four helical regions, of which two form coiledcoil assemblies. The carboxy-terminus constitutively binds the calcium sensor, calmodulin [94]. The mutation removes three helices, essentially abrogating calmodulin binding. The ENU12 strain has a phenotype similar to the complete knock-out demonstrating an essential role for the helical domain in KCNQ1 function.

ENU16/069 Pedigree (in Short: ENU16). With minimal stress, ENU16 mice display a mild action tremor. With moderate stress the tremor worsens and the mice display an unusual, hypermetric gait in their hind limbs. Upon restraining of the tail either one or both hind limbs show dramatic "clasping", and when returned to the cage, it takes some time for the mice to regain control over the limbs with what looks to be a sporadic and short-term lack of control of the hind limbs. Within seconds of being returned to the cage post restraint the gait appears to improve but can persist and be quite unsteady and "waddle-like". The phenotype is noticeable from 4 to 5 weeks of age.

Deep sequencing identified an A to G substitution of bp 530 in exon 5 of the Mpz (Myelin Protein Zero) gene resulting in an Asp to Gly change of aa 121. Aliases of MPZ are MPP, P-zero, and P0, among others. Schwann cells, the glia of the peripheral nervous system (PNS), play pivotal roles in the development and maintenance of the PNS. In addition to forming the myelin sheath, Schwann cells are involved in neuronal survival where they also provide support to axons during development and throughout adulthood. The $M p z$ gene encodes a transmembrane glycoprotein that is the major structural protein of peripheral myelin. Mutations in the $M p z$ gene result in the autosomal dominant form of Charcot-Marie-Tooth disease type 1 (CMT1B) [95]. Over 120 point mutations have been identified in humans until today (http://www.molgen.ua.ac.be/CMTMutations/Mutation/Default.cfm). The structure of the extracellular domain of P0 resembles an immunoglobulin variable domain. This domain is important for the homotypic interactions that convey the self-adhesive properties that are essential to maintain myelin compaction and integrity [96].

The complete P0 knockout causes a severe demyelinating neuropathy [96], but the function of P0 is still not fully resolved [97]. The mice are deficient in normal motor coordination and exhibit tremors and occasional convulsions. Axons in their peripheral nerves are severely hypomyelinated, and a subset contain myelin-like figures and axons degenerate. A second strain is available from Jackson Laboratories: B6.Cg-Mpz $z^{\text {ttrr }} / \mathrm{GrsrJ}$ mice carry the spontaneous totterer mutation. The underlying gene mutation has not been reported. Homozygous mice are poor breeders, reproducing at most once or twice, and as females are poor mothers, use of the strain is limited. There are also transgenic strains available that overexpress CMT1B mutant forms of $\mathrm{Mpz}$ [98]. How does the ENU16 strain differ from available mutant strains? Besides the totterer strain, for P0 there is only a complete knock-out available, and not a knock-in strain. The ENU16 strain has an N121G point mutation that is juxtaposed to the only known glycosylation site in the protein. We found however by immunohistochemistry and Western blot analysis that the mutated protein (P0 N121G) is expressed at a pattern and at levels similar to wild-type. This allows us to employ the ENU16 strain in studies (such as of adhesion) that are not feasible with the complete knockout.

\section{Outlook}

This overview of pedigrees with motor phenotypes reveals that mice are amenable to ENU mutagenesis and that it is possible to establish interesting phenotypes. Compared to the functional genomics approaches we have pursued in the past [99-102], ENU mutagenesis is more directed as it not only identifies gene mutations that modulate an existing pathology but also has the added advantage of establishing mutant mouse strains at the same time. As outlined above, ENU tends to introduce point mutations rather than causing deletions; this results in the expression of mutant proteins at levels that are high enough to perform functional studies. A limitation in a more wide-spread application of ENU mutagenesis though to mice is the high costs associated with establishing the mutants and identifying the underlying gene mutation and the fact that not all phenotypes are amenable to a robust and fast read-out. Therefore, using the motor phenotype as a surrogate marker for memory functions (as 
for the $\mathrm{K} 3$ transgene-dependent pedigrees) is an avenue that can be pursued in the field of AD mouse models.

What are the challenges of ENU mutagenesis in transgenic models of neurodegeneration and what issues both practical and conceptual did we encounter while doing these studies? In hindsight it appears to have been the preferred strategy to start with as many ENU-mutagenised males for cross-breeding with $\mathrm{K} 3$ mice as possible in order to saturate the genome with mutations. Also, we had initially planned to identify both dominant and recessive mutations, but considering the extensive breeding needed, we realised that such an undertaking is not affordable and therefore we concentrated solely on dominantly inherited traits. In principle however recessive mutations would be very interesting to work with.

The question that may be raised is which other transgenic models could be easily screened and used for ENU mutagenesis. We believe that any strain with a phenotype that can be easily picked up is amenable to this strategy. In our case we have been using mice that are characterized by a robust tau-dependent motor and memory phenotype, so we assumed that any modification of the motor phenotype would also modify memory functions, a trait, which would be tested after the modification of the motor phenotype has been confirmed. Another question is what the expectations are beyond finding possible new targets and how we see the field evolving in the years ahead. We have started to establish Caenorhabditis elegans in the laboratory as a system complementary to mice and it seems that overall a mutagenesis screen in the roundworm system is easier. We still believe that there is a potential in applying ENU mutagenesis to mouse models. These models might nicely complement ongoing concerted efforts such as those of the International Knockout Mouse Consortium (http://www.knockoutmouse.org/). The main objectives of this consortium are to generate, archive, and distribute worldwide up to 12.000 conditional mutations across the mouse genome in mouse embryonic stem (ES) cells. Another goal is to establish a limited number of mouse mutants from this resource. The main mutagenesis strategies pursued are: (i) conditional gene trapping-a random approach for expressed genes; (ii) conditional-targeted trapping - a directed approach, used for expressed genes; and (iii) conditional gene targeting - a directed approach, used for non-expressed genes

Hopefully, these concerted efforts help in developing a cure for a disease for which only symptomatic treatment is available [1].

\section{Acknowledgment}

This research was supported by grants to J. Götz from the ARC, the NHMRC, and the Judith Jane Mason \& Harold Stannett Williams Memorial Foundation, and to L. Ittner from the NHMRC and ARC.

\section{References}

[1] C. Ballard, S. Gauthier, A. Corbett et al., "Alzheimer's disease," The Lancet, vol. 377, no. 9770, pp. 1019-1031, 2011.
[2] R. A. Stelzmann, H. N. Schnitzlein, and F. R. Murtagh, "An English translation of Alzheimer's 1907 paper, 'uber eine eigenartige erkankung der hirnrinde," Clinical Anatomy, vol. 8, no. 6, pp. 429-431, 1995.

[3] F. Mangialasche, A. Solomon, B. Winblad, P. Mecocci, and M. Kivipelto, "Alzheimer's disease: clinical trials and drug development," The Lancet Neurology, vol. 9, no. 7, pp. 702716, 2010.

[4] F. Chen, D. David, A. Ferrari, and J. Götz, "Posttranslational modifications of tau-tole in human tauopathies and modeling in transgenic animals," Current Drug Targets, vol. 5, no. 6, pp. 503-515, 2004.

[5] L. M. Ittner, Y. D. Ke, F. Delerue et al., "Dendritic function of tau mediates amyloid- $\beta$ toxicity in Alzheimer's disease mouse models," Cell, vol. 142, no. 3, pp. 387-397, 2010.

[6] L. M. Ittner and J. Götz, "Amyloid- $\beta$ and tau-a toxic pas de deux in Alzheimer's disease," Nature Reviews Neuroscience, vol. 12, no. 2, pp. 65-72, 2011.

[7] D. J. Selkoe, "Alzheimer's disease is a synaptic failure," Science, vol. 298, no. 5594, pp. 789-791, 2002.

[8] M. Neumann, D. M. Sampathu, L. K. Kwong et al., "Ubiquitinated TDP-43 in frontotemporal lobar degeneration and amyotrophic lateral sclerosis," Science, vol. 314, no. 5796, pp. 130-133, 2006.

[9] Y. J. Zhang, Y. F. Xu, C. A. Dickey et al., "Progranulin mediates caspase-dependent cleavage of TAR DNA binding protein-43," Journal of Neuroscience, vol. 27, no. 39, pp. 10530-10534, 2007.

[10] L. M. Igaz, L. K. Kwong, A. Chen-Plotkin et al., "Expression of TDP-43 C-terminal fragments in vitro recapitulates pathological features of TDP-43 proteinopathies," Journal of Biological Chemistry, vol. 284, no. 13, pp. 8516-8524, 2009.

[11] D. Dormann, A. Capell, A. M. Carlson et al., "Proteolytic processing of TAR DNA binding protein- 43 by caspases produces C-terminal fragments with disease defining properties independent of progranulin," Journal of Neurochemistry, vol. 110, no. 3, pp. 1082-1094, 2009.

[12] H. Urwin, K. A. Josephs, J. D. Rohrer et al., "FUS pathology defines the majority of tau-and TDP-43-negative frontotemporal lobar degeneration," Acta Neuropathologica, vol. 120, no. 1, pp. 33-41, 2010.

[13] D. Dormann and C. Haass, "TDP-43 and FUS: a nuclear affair," Trends in Neurosciences, vol. 34, no. 7, pp. 339-348, 2011.

[14] L. Bertram and R. E. Tanzi, "Thirty years of Alzheimer's disease genetics: the implications of systematic meta-analyses," Nature Reviews Neuroscience, vol. 9, no. 10, pp. 768-778, 2008.

[15] A. D. Roses, "An inherited variable poly-T repeat genotype in TOMM40 in Alzheimer disease," Archives of Neurology, vol. 67, no. 5, pp. 536-541, 2010.

[16] J. C. Lambert, S. Heath, G. Even et al., "Genome-wide association study identifies variants at CLU and CR1 associated with Alzheimer's disease," Nature Genetics, vol. 41, no. 10, pp. 1094-1099, 2009.

[17] D. Harold, R. Abraham, P. Hollingworth et al., "Genomewide association study identifies variants at CLU and PICALM associated with Alzheimer's disease," Nature Genetics, vol. 41, no. 10, pp. 1088-1093, 2009.

[18] L. Jones, P. A. Holmans, M. L. Hamshere et al., "Genetic evidence implicates the immune system and cholesterol metabolism in the aetiology of Alzheimer's disease," PLoS One, vol. 5, no. 11, Article ID e13950, 2010. 
[19] I. R. A. Mackenzie, R. Rademakers, and M. Neumann, "TDP-43 and FUS in amyotrophic lateral sclerosis and frontotemporal dementia," The Lancet Neurology, vol. 9, no. 10, pp. 995-1007, 2010.

[20] V. M. Y. Lee, M. Goedert, and J. Q. Trojanowski, "Neurodegenerative tauopathies," Annual Review of Neuroscience, vol. 24, pp. 1121-1159, 2001.

[21] M. Cruts and C. Van Broeckhoven, "Loss of progranulin function in frontotemporal lobar degeneration," Trends in Genetics, vol. 24, no. 4, pp. 186-194, 2008.

[22] L. M. Ittner, T. Fath, Y. D. Ke et al., "Parkinsonism and impaired axonal transport in a mouse model of frontotemporal dementia," Proceedings of the National Academy of Sciences of the United States of America, vol. 105, no. 41, pp. 15997 16002,2008

[23] K. A. Nelms and C. C. Goodnow, "Genome-wide ENU mutagenesis to reveal immune regulators," Immunity, vol. 15, no. 3, pp. 409-418, 2001.

[24] J. Götz, "Tau and transgenic animal models," Brain Research Reviews, vol. 35, no. 3, pp. 266-286, 2001.

[25] J. Götz, J. R. Streffer, D. David et al., "Transgenic animal models of Alzheimer's disease and related disorders: histopathology, behavior and therapy," Molecular Psychiatry, vol. 9, no. 7, pp. 664-683, 2004.

[26] J. Götz, N. Deters, A. Doldissen et al., "A decade of tau transgenic animal models and beyond," Brain Pathology, vol. 17, no. 1, pp. 91-103, 2007.

[27] J. Götz and L. M. Ittner, "Animal models of Alzheimer's disease and frontotemporal dementia," Nature Reviews Neuroscience, vol. 9, no. 7, pp. 532-544, 2008.

[28] J. Gotz, A. Probst, M. G. Spillantini et al., "Somatodendritic localization and hyperphosphorylation of tau protein in transgenic mice expressing the longest human brain tau isoform," EMBO Journal, vol. 14, no. 7, pp. 1304-1313, 1995.

[29] A. Probst, M. Tolnay, C. Mistl et al., "Axonopathy and amyotrophy in mice transgenic for human four-repeat tau protein," Acta Neuropathologica, vol. 99, no. 5, pp. 469-481, 2000.

[30] J. Götz and R. M. Nitsch, "Compartmentalized tau hyperphosphorylation and increased levels of kinases in transgenic mice," NeuroReport, vol. 12, no. 9, pp. 2007-2016, 2001.

[31] J. Götz, F. Chen, R. Barmettler, and R. M. Nitsch, "Tau filament formation in transgenic mice expressing P301L tau," Journal of Biological Chemistry, vol. 276, no. 1, pp. 529-534, 2001.

[32] J. Götz, M. Tolnay, R. Barmettler, F. Chen, A. Probst, and R. M. Nitsch, "Oligodendroglial tau filament formation in transgenic mice expressing G272V tau," European Journal of Neuroscience, vol. 13, no. 11, pp. 2131-2140, 2001.

[33] J. Götz, F. Chen, J. Van Dorpe, and R. M. Nitsch, "Formation of neurofibrillary tangles in P301L tau transgenic mice induced by A $\beta 42$ fibrils," Science, vol. 293, no. 5534, pp. 14911495, 2001.

[34] L. Pennanen, H. Welzl, P. D’Adamo, R. M. Nitsch, and J. Götz, "Accelerated extinction of conditioned taste aversion in P301L tau transgenic mice," Neurobiology of Disease, vol. 15, no. 3, pp. 500-509, 2004.

[35] L. Pennanen, D. P. Wolfer, R. M. Nitsch, and J. Götz, "Impaired spatial reference memory and increased exploratory behavior in P301L tau transgenic mice," Genes, Brain and Behavior, vol. 5, no. 5, pp. 369-379, 2006.

[36] N. Deters, L. M. Ittner, and J. Götz, "Divergent phosphorylation pattern of tau in P301L tau transgenic mice," European Journal of Neuroscience, vol. 28, no. 1, pp. 137-147, 2008.
[37] N. Deters, L. M. Ittner, and J. Götz, "Substrate-specific reduction of PP2A activity exaggerates tau pathology," Biochemical and Biophysical Research Communications, vol. 379, no. 2, pp. 400-405, 2009.

[38] C. Köhler, P. Bista, J. Götz, and H. Schröder, "Analysis of the cholinergic pathology in the P301L tau transgenic pR5 model of tauopathy," Brain Research, vol. 1347, pp. 111-124, 2010.

[39] D. C. David, L. M. Ittner, P. Gehrig et al., " $\beta$-amyloid treatment of two complementary P301L tau-expressing Alzheimer's disease models reveals similar deregulated cellular processes," Proteomics, vol. 6, no. 24, pp. 6566-6577, 2006.

[40] D. C. David, S. Hauptmann, I. Scherping et al., "Proteomic and functional analyses reveal a mitochondrial dysfunction in P301L tau transgenic mice," Journal of Biological Chemistry, vol. 280, no. 25, pp. 23802-23814, 2005.

[41] A. Eckert, S. Hauptmann, I. Scherping et al., "Oligomeric and fibrillar species of $\beta$-amyloid (A $\beta 42)$ both impair mitochondrial function in P301L tau transgenic mice," Journal of Molecular Medicine, vol. 86, no. 11, pp. 1255-1267, 2008.

[42] A. Eckert, S. Hauptmann, I. Scherping et al., "Soluble betaamyloid leads to mitochondrial defects in amyloid precursor protein and tau transgenic mice," Neurodegenerative Diseases, vol. 5, no. 3-4, pp. 157-159, 2008.

[43] N. Schonrock, Y. D. Ke, D. Humphreys et al., "Neuronal microRNA deregulation in response to Alzheimer's disease amyloid-beta," PloS One, vol. 5, no. 6, p. e11070, 2010.

[44] N. Schonrock, D. T. Humphreys, T. Preiss, and J. Götz, "Target Gene Repression Mediated by miRNAs miR-181c and miR-9 Both of Which Are Down-regulated by Amyloid- $\beta$," Journal of Molecular Neuroscience. In press.

[45] M. Neumann, W. Schulz-Schaeffer, R. Anthony Crowther et al., "Pick's disease associated with the novel Tau gene mutation K369I," Annals of Neurology, vol. 50, no. 4, pp. 503513, 2001.

[46] G. B. Stokin, C. Lillo, T. L. Falzone et al., "Axonopathy and transport deficits early in the pathogenesis of Alzheimer's diseases," Science, vol. 307, no. 5713, pp. 1282-1288, 2005.

[47] J. Götz, L. M. Ittner, and S. Kins, "Do axonal defects in tau and amyloid precursor protein transgenic animals model axonopathy in Alzheimer's disease?" Journal of Neurochemistry, vol. 98, no. 4, pp. 993-1006, 2006.

[48] E. Magnani, J. Fan, L. Gasparini et al., "Interaction of tau protein with the dynactin complex," EMBO Journal, vol. 26, no. 21, pp. 4546-4554, 2007.

[49] E. Thies and E. M. Mandelkow, "Missorting of tau in neurons causes degeneration of synapses that can be rescued by the kinase MARK2/Par-1," Journal of Neuroscience, vol. 27, no. 11, pp. 2896-2907, 2007.

[50] R. Dixit, J. L. Ross, Y. E. Goldman, and E. L. F. Holzbaur, "Differential regulation of dynein and kinesin motor proteins by tau," Science, vol. 319, no. 5866, pp. 1086-1089, 2008.

[51] G. Pigino, G. Morfini, Y. Atagi et al., "Disruption of fast axonal transport is a pathogenic mechanism for intraneuronal amyloid beta," Proceedings of the National Academy of Sciences of the United States of America, vol. 106, no. 14, pp. 59075912, 2009.

[52] J. Gotz, A. Eckert, M. Matamales, L.M. Ittner, and X. Liu, "Modes of A beta toxicity in Alzheimer's disease," Cellular and Molecular Life Sciences, vol. 68, no. 20, pp. 3359-3375, 2011.

[53] B. Su, X. Wang, A. Nunomura et al., "Oxidative stress signaling in Alzheimer's disease," Current Alzheimer Research, vol. 5, no. 6, pp. 525-532, 2008. 
[54] T. Tatsuta and T. Langer, "Quality control of mitochondria: protection against neurodegeneration and ageing," $E M B O$ Journal, vol. 27, no. 2, pp. 306-314, 2008.

[55] L. M. Ittner, Y. D. Ke, and J. Götz, "Phosphorylated Tau interacts with $\mathrm{c}$-Jun $\mathrm{N}$-terminal kinase-interacting protein 1 (JIP1) in Alzheimer disease," Journal of Biological Chemistry, vol. 284, no. 31, pp. 20909-20916, 2009.

[56] J. Götz, A. Ittner, and L. M. Ittner, "Tau-targeted treatment strategies in Alzheimer's disease," British Journal of Pharmacology. In press.

[57] J. Van Eersel, Y. D. Ke, X. Liu et al., "Sodium selenate mitigates tau pathology, neurodegeneration, and functional deficits in Alzheimer's disease models," Proceedings of the $\mathrm{Na}$ tional Academy of Sciences of the United States of America, vol. 107, no. 31, pp. 13888-13893, 2010.

[58] Y. D. Ke, F. Delerue, A. Gladbach, J. Götz, and L. M. Ittner, "Experimental diabetes mellitus exacerbates Tau pathology in a transgenic mouse model of Alzheimer's disease," PLoS One, vol. 4, no. 11, Article ID e7917, 2009.

[59] A. Ferrari, E. Ehler, R. M. Nitsch, and J. Götz, "Immature human NT2 cells grafted into mouse brain differentiate into neuronal and glial cell types," FEBS Letters, vol. 486, no. 2, pp. 121-125, 2000.

[60] M. Blurton-Jones, M. Kitazawa, H. Martinez-Coria et al., "Neural stem cells improve cognition via BDNF in a transgenic model of Alzheimer disease," Proceedings of the National Academy of Sciences of the United States of America, vol. 106, no. 32, pp. 13594-13599, 2009.

[61] H. Rosenmann, N. Grigoriadis, D. Karussis et al., "Tauopathy-like abnormalities and neurologic deficits in mice immunized with neuronal tau protein," Archives of Neurology, vol. 63, no. 10, pp. 1459-1467, 2006.

[62] A. A. Asuni, A. Boutajangout, D. Quartermain, and E. M. Sigurdsson, "Immunotherapy targeting pathological tau conformers in a tangle mouse model reduces brain pathology with associated functional improvements," Journal of Neuroscience, vol. 27, no. 34, pp. 9115-9129, 2007.

[63] A. Boutajangout, D. Quartermain, and E. M. Sigurdsson, "Immunotherapy targeting pathological tau prevents cognitive decline in a new tangle mouse model," Journal of Neuroscience, vol. 30, no. 49, pp. 16559-16566, 2010.

[64] M. Boimel, N. Grigoriadis, A. Lourbopoulos, E. Haber, O. Abramsky, and H. Rosenmann, "Efficacy and safety of immunization with phosphorylated tau against neurofibrillary tangles in mice," Experimental Neurology, vol. 224, no. 2, pp. 472-485, 2010.

[65] J. Gotz, A. Eckert, M. Matamales, L.M. Ittner, and X. Liu, "Modes of A beta toxicity in Alzheimer's disease," Cellular and Molecular Life Sciences, vol. 68, no. 20, pp. 3359-3375, 2011.

[66] A. Schild, S. Isenmann, N. Tanimoto et al., "Impaired development of the Harderian gland in mutant protein phosphatase 2A transgenic mice," Mechanisms of Development, vol. 123 , no. 5, pp. 362-371, 2006.

[67] A. Schild, L. M. Ittner, and J. Götz, "Altered phosphorylation of cytoskeletal proteins in mutant protein phosphatase $2 \mathrm{~A}$ transgenic mice," Biochemical and Biophysical Research Communications, vol. 343, no. 4, pp. 1171-1178, 2006.

[68] T. Takeda, "Senescence-accelerated mouse (SAM) with special references to neurodegeneration models, SAMP8 and SAMP10 mice," Neurochemical Research, vol. 34, no. 4, pp. 639-659, 2009.

[69] M. C. Cook, C. G. Vinuesa, and C. C. Goodnow, "ENUmutagenesis: insight into immune function and pathology,"
Current Opinion in Immunology, vol. 18, no. 5, pp. 627-633, 2006.

[70] S. Flibotte, M. L. Edgley, I. Chaudhry et al., "Whole-genome profiling of mutagenesis in Caenorhabditis elegans," Genetics, vol. 185, no. 2, pp. 431-441, 2010.

[71] C. Nuesslein-Volhard, M. Lohs-Schardin, K. Sander, and C. Cremer, "A dorso-ventral shift of embryonic primordia in a new maternal-effect mutant of Drosophila," Nature, vol. 283, no. 5746, pp. 474-476, 1980.

[72] S. Hitotsumachi, D. A. Carpenter, and W. L. Russel, "Doserepetition increases the mutagenic effectiveness of N-ethyl$\mathrm{N}$-nitrosourea in mouse spermatogonia," Proceedings of the National Academy of Sciences of the United States of America, vol. 82, no. 19, pp. 6619-6621, 1985.

[73] A. Theodoratos, B. Whittle, A. Enders et al., "Mouse strains with point mutations in TAP1 and TAP2," Immunology and Cell Biology, vol. 88, no. 1, pp. 72-78, 2010.

[74] J. Chu, N. A. Hong, C. A. Masuda et al., "A mouse forward genetics screen identifies LISTERIN as an E3 ubiquitin ligase involved in neurodegeneration," Proceedings of the National Academy of Sciences of the United States of America, vol. 106, no. 7, pp. 2097-2103, 2009.

[75] E. L. Coghill, A. Hugill, N. Parkinson et al., "A gene-driven approach to the identification of ENU mutants in the mouse," Nature Genetics, vol. 30, no. 3, pp. 255-256, 2002.

[76] L. B. Barnett, R. W. Tyl, B. S. Shane, M. D. Shelby, and S. E. Lewis, "Transmission of mutations in the lacI transgene to the offspring of ENU-treated Big Blue male mice," Environmental and Molecular Mutagenesis, vol. 40, no. 4, pp. 251257, 2002.

[77] B. T. Kile, K. E. Hentges, A. T. Clark et al., "Functional genetic analysis of mouse chromosome 11," Nature, vol. 425, no. 6953, pp. 81-86, 2003.

[78] D. Concepcion, K. L. Seburn, G. Wen, W. N. Frankel, and B. A. Hamilton, "Mutation rate and predicted phenotypic target sizes in ethylnitrosourea- treated mice," Genetics, vol. 168, no. 2, pp. 953-959, 2004.

[79] M. M. Quwailid, A. Hugill, N. Dear et al., "A gene-driven ENU-based approach to generating an allelic series in any gene," Mammalian Genome, vol. 15, no. 8, pp. 585-591, 2004.

[80] Y. Sakuraba, H. Sezutsu, K. R. Takahasi et al., "Molecular characterization of ENU mouse mutagenesis and archives," Biochemical and Biophysical Research Communications, vol. 336, no. 2, pp. 609-616, 2005.

[81] M. J. Justice, J. K. Noveroske, J. S. Weber, B. Zheng, and A. Bradley, "Mouse ENU mutagenesis," Human Molecular Genetics, vol. 8, no. 10, pp. 1955-1963, 1999.

[82] B. T. Kile and D. J. Hilton, "The art and design of genetic screens: mouse," Nature Reviews Genetics, vol. 6, no. 7, pp. 557-567, 2005.

[83] P. M. Nolan, J. Peters, M. Strivens et al., "A systematic, genome-wide, phenotype-driven mutagenesis programme for gene function studies in the mouse," Nature Genetics, vol. 25, no. 4, pp. 440-443, 2000.

[84] R. T. Durrett, K. Y. Chen, and S. D. Tanksley, "A simple formula useful for positional cloning," Genetics, vol. 160, no. 1, pp. 353-355, 2002.

[85] B. J. Herron, W. Lu, C. Rao et al., "Efficient generation and mapping of recessive developmental mutations using ENU mutagenesis," Nature Genetics, vol. 30, no. 2, pp. 185-189, 2002.

[86] M. L. Metzker, "Sequencing technologies the next generation," Nature Reviews Genetics, vol. 11, no. 1, pp. 31-46, 2010. 
[87] L. A. Jerome and V. E. Papaioannou, "DiGeorge syndrome phenotype in mice mutant for the T-box gene, Tbx1," Nature Genetics, vol. 27, no. 3, pp. 286-291, 2001.

[88] J. M. Long, P. LaPorte, S. Merscher et al., "Behavior of mice with mutations in the conserved region deleted in velocardiofacial/DiGeorge syndrome," Neurogenetics, vol. 7, no. 4, pp. 247-257, 2006.

[89] H. Wurdak, L. M. Ittner, and L. Sommer, "DiGeorge syndrome and pharyngeal apparatus development," BioEssays, vol. 28, no. 11, pp. 1078-1086, 2006.

[90] L. N. Kong, P. P. Zuo, L. Mu, Y. Y. Liu, and N. Yang, "Gene expression profile of amyloid beta protein-injected mouse model for Alzheimer disease," Acta Pharmacologica Sinica, vol. 26, no. 6, pp. 666-672, 2005.

[91] A. Jervell and F. Lange-Nielsen, "Congenital deaf-mutism, functional heart disease with prolongation of the Q-T interval, and sudden death," American Heart Journal, vol. 54, no. 1, pp. 59-68, 1957.

[92] M. C. Casimiro, B. C. Knollmann, S. N. Ebert et al., "Targeted disruption of the Kcnq1 gene produces a mouse model of Jervell and Lange-Nielsen Syndrome," Proceedings of the National Academy of Sciences of the United States of America, vol. 98, no. 5, pp. 2526-2531, 2001.

[93] M. C. Casimiro, B. C. Knollmann, E. N. Yamoah et al., “Targeted point mutagenesis of mouse Kcnq1: phenotypic analysis of mice with point mutations that cause Romano-Ward syndrome in humans," Genomics, vol. 84, no. 3, pp. 555-564, 2004.

[94] R. Wiener, Y. Haitin, L. Shamgar et al., “The KCNQ1 (Kv7.1) $\mathrm{COOH}$ terminus, a multitiered scaffold for subunit assembly and protein interaction," Journal of Biological Chemistry, vol. 283, no. 9, pp. 5815-5830, 2008.

[95] M. E. Shy, "Peripheral neuropathies caused by mutations in the myelin protein zero," Journal of the Neurological Sciences, vol. 242, no. 1-2, pp. 55-66, 2006.

[96] K. P. Giese, R. Martini, G. Lemke, P. Soriano, and M. Schachner, "Mouse P0 gene disruption leads to hypomyelination, abnormal expression of recognition molecules, and degeneration of myelin and axons," Cell, vol. 71, no. 4, pp. 565-576, 1992.

[97] B. Ey, I. Kobsar, H. Blazyca, A. Kroner, and R. Martini, "Visualization of degenerating axons in a dysmyelinating mouse mutant with axonal loss," Molecular and Cellular Neuroscience, vol. 35, no. 1, pp. 153-160, 2007.

[98] A. E. Rünker, I. Kobsar, T. Fink et al., "Pathology of a mouse mutation in peripheral myelin protein $\mathrm{P} 0$ is characteristic of a severe and early onset form of human Charcot-Marie-Tooth type 1B disorder," Journal of Cell Biology, vol. 165, no. 4, pp. 565-573, 2004.

[99] F. J. Hoerndli, M. Toigo, A. Schild, J. Götz, and P. J. Day, "Reference genes identified in SH-SY5Y cells using custom-made gene arrays with validation by quantitative polymerase chain reaction," Analytical Biochemistry, vol. 335, no. 1, pp. 30-41, 2004.

[100] F. Hoerndli, D. C. David, and J. Götz, "Functional Genomics meets neurodegenerative disorders: part II: application and data integration," Progress in Neurobiology, vol. 76, no. 3, pp. 169-188, 2005.

[101] D. C. David, F. Hoerndli, and J. Götz, "Functional Genomics meets neurodegenerative disorders: part I: transcriptomic and proteomic technology," Progress in Neurobiology, vol. 76, no. 3, pp. 153-168, 2005.

[102] I. S. Pienaar, W. M. U. Daniels, and J. Götz, "Neuroproteomics as a promising tool in Parkinson's disease research,"
Journal of Neural Transmission, vol. 115, no. 10, pp. 14131430, 2008. 

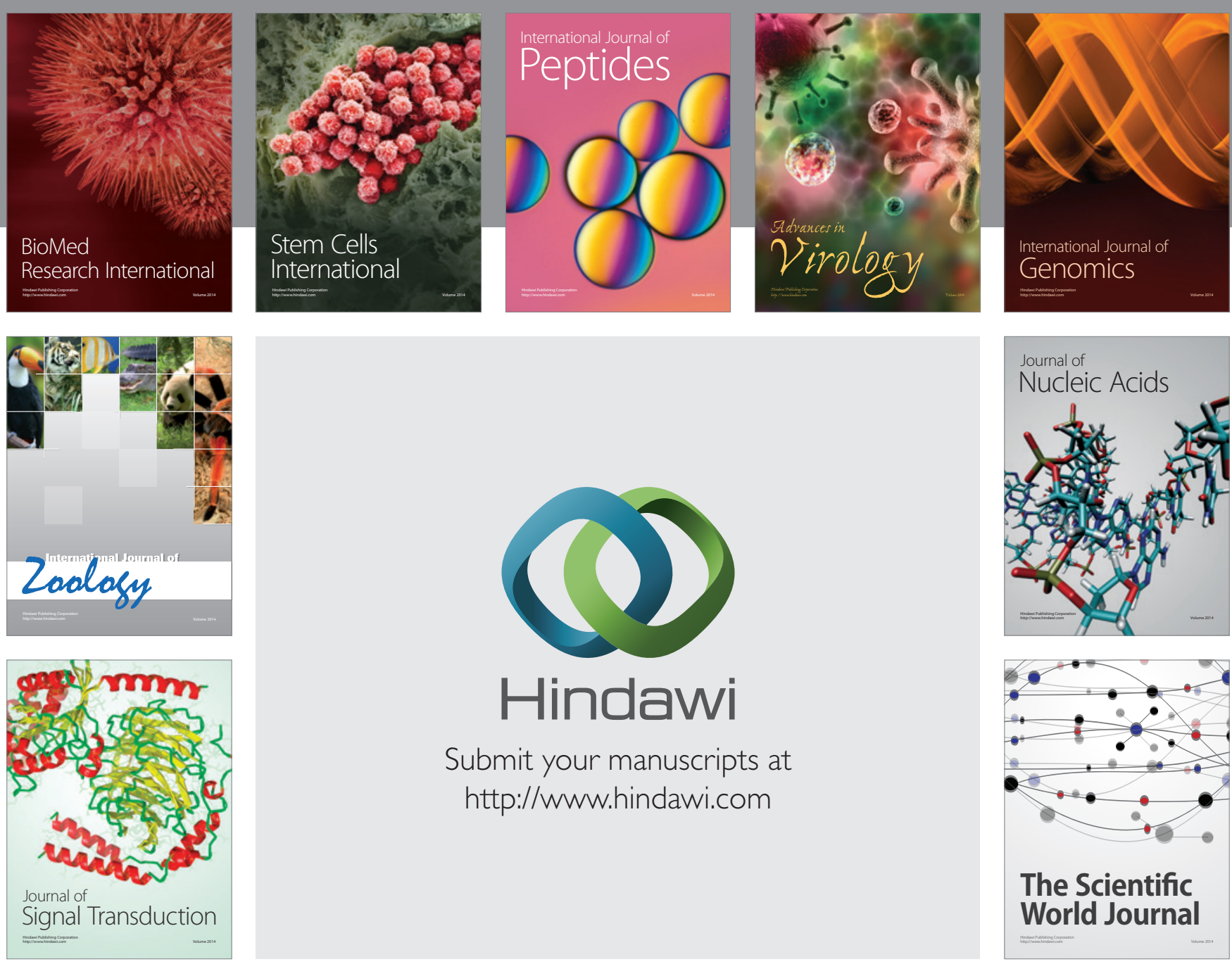

Submit your manuscripts at

http://www.hindawi.com
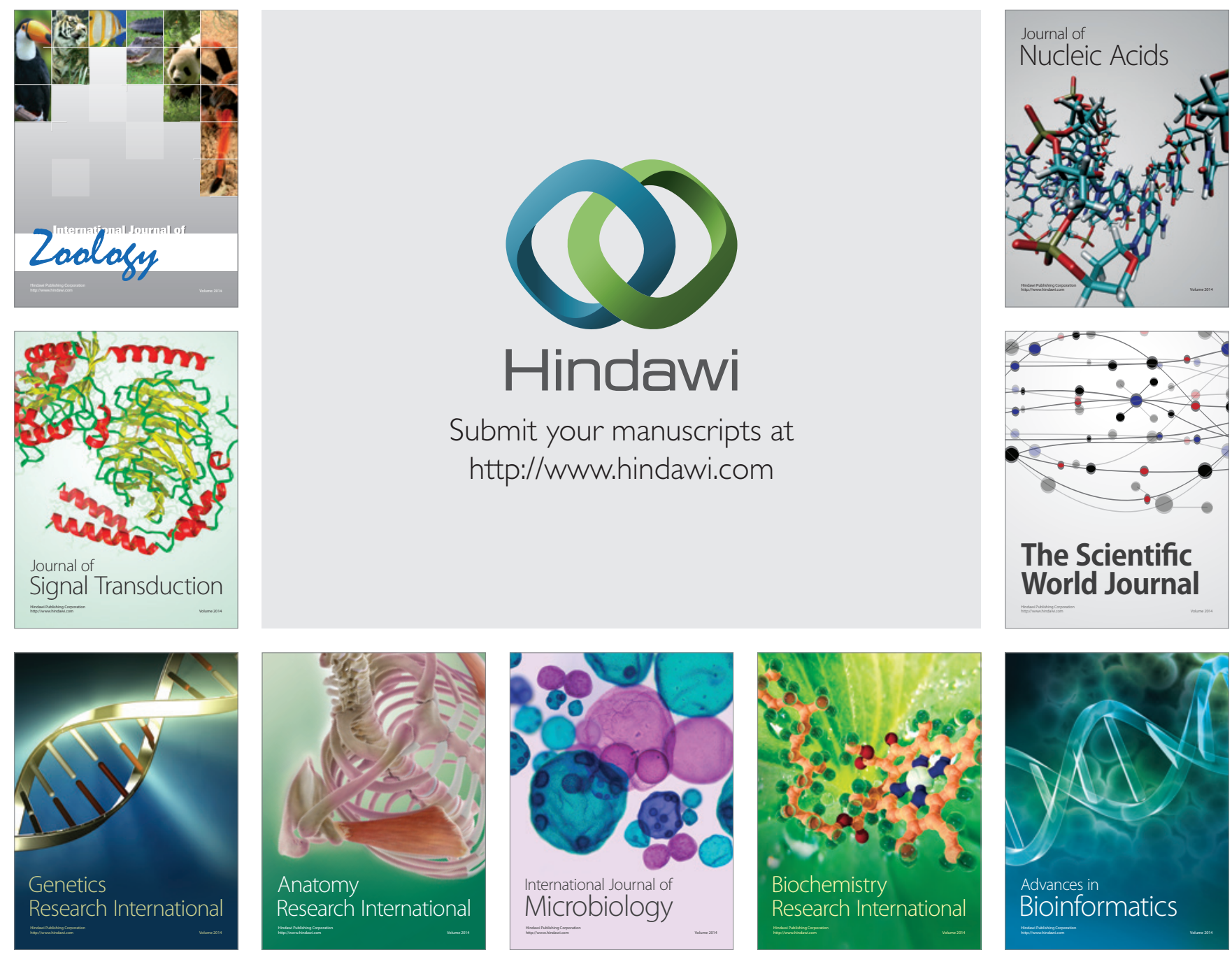

The Scientific World Journal
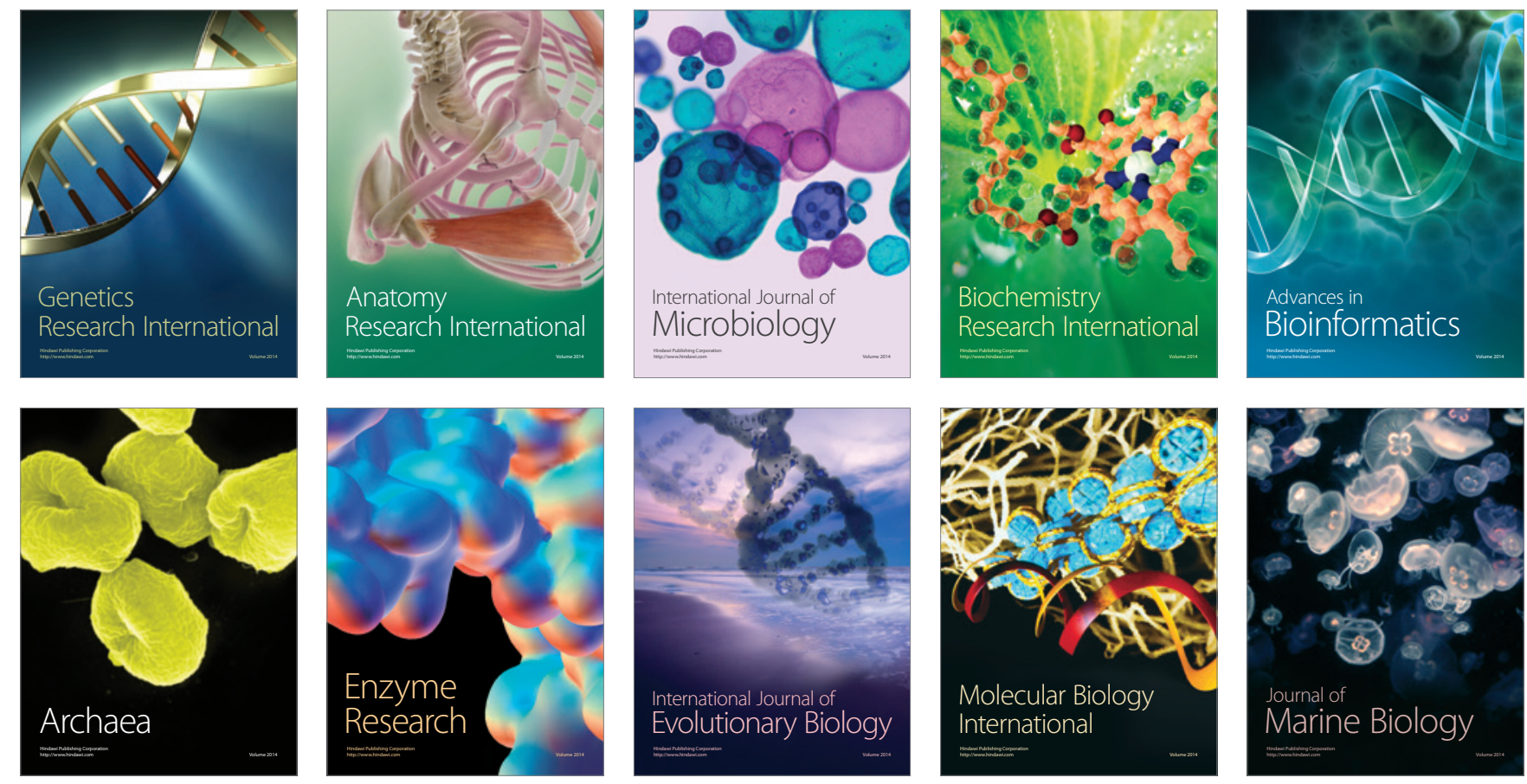